\title{
Pattern of Antibiotic Resistance in Salmonella Typhi Isolates with Special Consideration to Extended Drug Resistant Typhoid
}

Ashfaq Ahmad, Salman Mustaan, Fazal Wahab, Muhammad Ayyaz, Abubakar Sadiq, Sara, ljaz Hussain

\section{ABSTRACT}

Background: Typhoid or enteric fever is potentially fatal multisystem illness caused by salmonella typhi and Para typhi transmitted through consumption of contaminated food and water. Salmonella behave in a wide spectrum of resistance from Multi Drug Resistant typhoid to now Extended Drug Resistant typhoid.

Objective: To analyze the culture, sensitivity and resistance of salmonella typhi in patients with clinical suspicion of typhoid fever. Material and methods: A cross sectional study was performed on 624 clinically suspected cases of typhoid fever admitted to the inpatient ward facility of Pediatric unit STH Swat from $1^{\text {st }}$ July 2019 to $30^{\text {th }}$ June 2020. Data was analyzed using SPSS version 22. Results: Out of 624 clinically suspected cases of typhoid fever reported to the Pediatric unit of STH Swat, only 239 cases had a positive blood culture for salmonella typhi during the period of 1 year. Drug resistance pattern of salmonella typhi in our study was ceftriaxone $91.86 \%$, chloramphenicol $83.72 \%$ ampicillin $75.58 \%$, co-trimoxazole $72.09 \%$, Quinolones $83.72 \%$, azithromycin $55.81 \%$, Cefoperazone/Sulbactum $77.9 \%$, Piperacillin/Tazobactam $65.11 \%$, and meropenem was low at $4.65 \%$ and no resistance was reported to imipenem.

Conclusion: In the light of our study the highest resistance of salmonella specie and emergence of XDR typhoid to the i.e. $3^{\text {rd }}$ generation cephalosporins and Azithromycin, it is necessary to rationalize the use of antibiotics in acute febrile illness and to educate the community for vaccination against salmonella typhi, proper sanitation and use of safe water supply . Blood culture should be the gold standard test for diagnosis of enteric fever in the first week of illness.

Key words: Salmonella typhi, XDR-typhoid

This article may be cited as: Ahmad A, Mustaan S, Wahab F, Ayyaz M, Sadiq A, Sara, Hussain I. Pattern of Antibiotic Resistance in Salmonella Typhi Isolates with special consideration to Extended Drug Resistant Typhoid. J Saidu Med Coll Swat 2021;11(2):103-107

\section{INTRODUCTION}

Infectious diseases possess a major health issue worldwide. Salmonellosis, a common food and water born infectious disease is caused by two different gram-negative bacteria i.e. Salmonella typhi (S. typhi) and Salmonella Para typhi (S. para typhi) ${ }^{1}$. Enteric fever caused by bacteria is transmitted through feco-oral route by ingestion of bacteria in contaminated food or water. After ingestion, it evades the low $\mathrm{PH}$ of the stomach and invades the local lymphatic tissues and peyers patches and get disseminated through the reticuloendothelial system causing the clinical syndrome in the form of variety of signs and symptoms ${ }^{2}$.

Around the world, it is a major health problem as enteric fever is endemic in many areas of the developing world. The organism infects millions of people worldwide and causes significant morbidity and mortality. According to a report from 2017 up to 15 million people were infected with the salmonella species and had enteric fever. Worldwide more than one lac patients died due to enteric fever and majority of the deaths were reported from Asia i.e. $69.9 \%{ }^{3}$. In a study

\section{Department of Peads, Saidu Group of Teaching Hospital, Saidu Sharif, Swat}

Correspondence: Dr. Muhammad Ayyaz

Department of Peads, Saidu Group of Teaching Hospital,

Saidu Sharif, Swat. ayyazaazee120@gmail.com comparing south Asian countries for prevalence of typhoid fever Pakistan had a very high burden of disease and majority of the effected population were pediatric age group i.e. below 15 years of age $^{4}$.

Proven and identified risk factors for enteric fever are the use of contaminated drinking water or food with faeces from either acutely ill patients, chronic asymptomatic carriers, poor sanitation system, improper hygienic practices, improper waste disposal and low socio-economic status ${ }^{5}$. Enteric fever is a serious systemic illness and needs prompt investigation and proper immediate management. Antimicrobial therapy is the mainstay in the management of enteric fever to limit morbidity and mortality due to it ${ }^{6}$.

The horrific resistance story of salmonella goes back to the early 1970s when for the first-time resistance to the then first line treatment chloramphenicol was documented and then later resistance to ampicillin and clotrimazole was also reported $^{7}$. Frequent epidemics of Multi drug resistant (MDR) salmonella since 1990 created a room for the use of fluroquinolones as the first line therapy. But in early 2000 due to frequent and over the counter use of ciprofloxacin for the treatment of enteric fever caused increase in resistance to it $^{8}$. 
Now the situation is worst as the resistance pattern of salmonella is widening its horizons to $3^{\text {rd }}$ generation cephalosporins and to azithromycin creating extended drug resistance (XDR) typhoid. In 2006, Aga Khan University reported MDR typhoid of $48.5 \%$ and resistance to quinolones to $64.1 \%{ }^{9}$. Up to the mid of this decade, cefixime and ceftriaxone were considered safe in treating uncomplicated and complicated typhoid fever respectively. The trend and situations are changed, and clinician are uncertain due the increasing resistance of the salmonella species to the $3^{\text {rd }}$ generation cephalosporins ${ }^{10}$.MDR and XDR typhoid has now become a serious threat as the bacteria is now infecting people in higher rate throughout the globe, causing serious and difficult to treat infections. Due to the emergence of resistance of the salmonella typhi to fluoroquinolones and third generation cephalosporins, now it is necessary to treat all patients with enteric fever based on its culture and sensitivity pattern ${ }^{11}$.

To estimate the burden of XDR typhoid, it is the need of the hour to screen the high-risk population for it. And in the initial phase of illness, blood culture is the only gold standard method for diagnosing enteric fever and assessing antibiotic resistance pattern. Blood culture is the very effective method of finding the prevalence of enteric fever in a given population and is used in surveillance programs for understanding burden of disease in various countries. These study programs aim to evaluate the incidence of disease by identify all cases of blood culture-positive typhoid fever in a population ${ }^{12}$.

In this study we planned to determine the antimicrobial drug resistance pattern in salmonella typhi. XDR typhoid is on the rise worldwide and it is the most threatening issue in infection control; therefor it is of prime importance to document its spread and pattern of antibiotic resistance in various regions.

\section{MATERIALS AND METHODS}

A cross sectional study was performed on 624 clinically suspected cases of typhoid fever admitted to the inpatient ward facility of Pediatric unit Saidu Teaching Hospital, Swat over the period of one year from $1^{\text {st }}$ July 2019 to $30^{\text {th }}$ June 2020 . Saidu Teaching Hospital is a tertiary care hospital located in Mingora district Swat. Pediatric unit STH is a referral center for many patients from inside the district of Swat along with other districts of Malakand division like Shangla, Buner,

\section{Kohistan and Dir.}

Sample size was estimated to be 214 and calculated through Open epi sample size calculator as the prevalence of culture proven enteric fever was $13 \%{ }^{9}$ and the confidence interval was $95 \%$. After proper written and verbal consent from the legal attendants of the patients $5 \mathrm{ml}$ of venous blood sample was collected through proper aseptic measures and the blood was inoculated in blood culture bottles i.e. Tryptone soya broth (TSB with SPS) from all the included patient. Upon growth in the blood culture bottle the samples were further inoculated in MacConkey agar plates. The antimicrobial sensitivity of Salmonella isolates were then tested by KirbyBauer disk diffusion technique on Mueller-Hinton agar (Oxide England). Antibiotic sensitivity tests were done for 10 antimicrobial drugs i.e. ampicillin, chloramphenicol, Co-Trimoxazole, Ciprofloxacin, Ceftriaxone, Azithromycin, Piperacilin-tazobactum, Cefoperazonesulbactam, Meropenem and Imipenem. Patients were treated on empiric basis first according to the guidelines and then culture reports were followed for further treatment and management

This study was approved by the ethical committee of Saidu Teaching Swat.

Inclusion Criteria: Patient having high grade fever for more than 5 days without localizing source of infection and having any of the clinical signs and symptoms suggesting of enteric fever like coated tongue, anorexia, vomiting, diarrhea, abdominal pain, pallor, headache, hepatomegaly, splenomegaly.

Exclusion criteria: All Patients having history of fever more than 5 days but presented with obvious signs and symptoms to upper and lower respiratory tract infections, Otitis media or blood culture negative for salmonella typhi.

\section{DEFINITIONS}

Multidrug resistant typhoid (MDR): Salmonella typhi isolates are fully resistant to first line antibiotics i.e. amoxicillin, chloramphenicol and trimethoprim-sulfamethoxazole ${ }^{13}$.

Extended drug resistant typhoid (XDR): Salmonella typhi isolates are fully resistant to all the antibiotics recommended for treating typhoid fever i.e. first- and second-line drugs ${ }^{13}$.

Data analysis: Data was analyzed in statistical package for social sciences version 22 (SPSS 22). Frequency and proportions were calculated for categorical variables while mean and standard deviation were taken for continuous variables. 
Pattern of Antibiotic Resistance in Salmonella Typhi Isolates with special consideration to Extended...

Pattern of drug resistance in salmonella typhi was compared using chi square test among different age groups, socioeconomic and geographic distribution and taken significant for $p$ value of $<$ 0.05 .

\section{RESULTS}

Out of 624 clinically suspected cases of typhoid fever reported to the Pediatric unit of STH Swat during the period of one year, only 239 cases had a positive blood culture for salmonella typhi. Culture positive typhoid fever was $38.3 \%$ of the total clinically suspected patients. No growth of $S$. para typhiwas reported.

The total positive blood culture typhoid fever patients, $62.3 \%$ were male and $37.6 \%$ were female. In the age group below 5 years, $21.33 \%$ of the total patients were male, $9.2 \%$ were female. In the age group between $5-10$ years, $19.24 \%$ of the total patients were male, $14.64 \%$ were female. In the age group $10-15$ years $12.55 \%$ of total patients were male, $7.53 \%$ were female. In the age group $>15$ years $9.2 \%$ of the total patients were male, $6.27 \%$ were female. The most common affected age group $(64.43 \%)$ included children below 10 years of age (Table 2 ).

Demographically, the patients were from different areas, i.e., $60.2 \%$ from Swat, $14.2 \%$ from Shangla, 9.2\% from Buner, 6.69\% from Kohistan, $9.6 \%$ from Dir. Majority of the XDR typhoid fever cases $71.42 \%$ were from district swat. The prevalence of typhoid fever was $33.4 \%$ of the patients were admitted from the months of July 2019 to September 2019 and 35.8\% from April 2020 to June 2020.

Majority of the children had a previous trial of antibiotics $(95.3 \%)$ and only $36.5 \%$ of the medicines were prescribed by a physician. The common drug used was cefixime $(43.9 \%)$ followed by ciprofloxacin (34.7\%) and ceftriaxone (21.4\%). $79.7 \%$ of the patient had no proper sanitation. $73.9 \%$ had low socioeconomic status. $94.3 \%$ of the patients had poor family background and only $5.7 \%$ of the patients had good hygienic practices. $46.83 \%$ patients had used spring water without boiling, $32.3 \%$ had used municipal water supply without boiling, $18.4 \%$ had used well water without boiling and only $2.47 \%$ of the patients had used filtered or boiled water. None of the patients in our study had received prior vaccination against salmonella typhi.

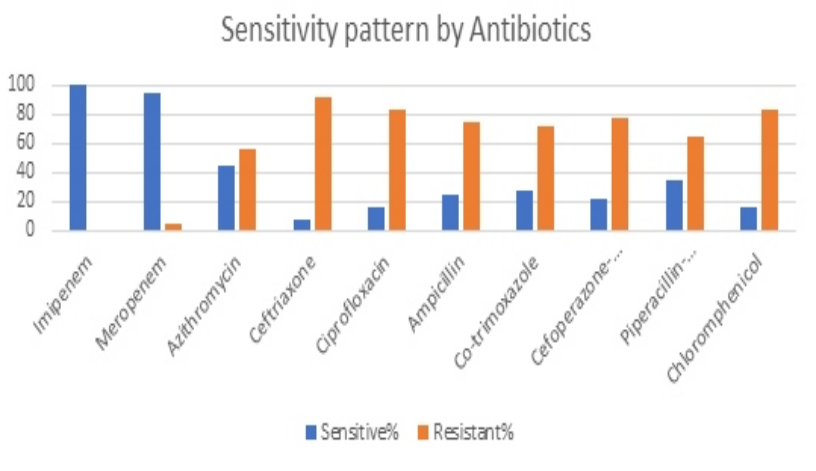

Figure1: Sensitivity pattern by antibiotics

Table 1: Salmonella typhi sensitivity and resistance pattern

\begin{tabular}{|c|c|c|}
\hline Drugs & Sensitive (\%) & Resistant (\%) \\
\hline Imipenem & 100 & 0 \\
\hline Meropenem & 95.35 & 4.65 \\
\hline Azithromycin & 44.19 & 55.81 \\
\hline Ceftriaxone & 8.14 & 91.86 \\
\hline Ciprofloxacin & 16.28 & 83.72 \\
\hline Ampicillin & 24.42 & 75.58 \\
\hline Co-Trimoxazole & 27.91 & 72.09 \\
\hline Cefoperazone-sulbactam & 22.1 & 77.9 \\
\hline Piperacilin-tazobactum & 34.89 & 65.11 \\
\hline Chloromphenicol & 16.28 & 83.72 \\
\hline
\end{tabular}

Table 2: Age and Gender wise distribution

\begin{tabular}{|c|c|c|c|}
\hline Age group & Male & Female & XDR-Typhoid \\
\hline$<5$ years & $21.33 \%$ & $9.2 \%$ & $18.79 \%$ \\
\hline 5 to 10 years & $19.24 \%$ & $14.64 \%$ & $36.09 \%$ \\
\hline 10 to 15 years & $12.55 \%$ & $7.53 \%$ & $20.3 \%$ \\
\hline$>15$ years & $9.2 \%$ & $156.27 \%$ & $24.81 \%$ \\
\hline
\end{tabular}


Drug resistance pattern of salmonella typhi to the first line drugs was very high in our study as compared to other studies i.e. chloramphenicol $83.72 \%$, ampicillin $75.58 \%$, co-trimoxazole $72.09 \%$.Resistance to other drugs like Cefoperazone/Sulbactum was $77.90 \%$, Piperacillin/Tazobactam 65.11\%, Quinolones resistance was $83.72 \%$ for ciprofloxacin which is another alarming sign. One of the concerning points was the prevalence of resistance to ceftriaxone that was $91.86 \%$ which is much high and represents the burden of XDR-typhoid. The resistance to azithromycin was also recorded very high $(55.81 \%)$. It may be due to the over counter frequent use of this drugs in upper and lower respiratory tract infection and sub therapeutic doses. Resistance to meropenem was low at $4.65 \%$ and no resistance was reported to imipenem (Table 1, Figure 1).

XDR-Typhoid was $55.81 \%$ of the total culture positive cases of which $18.79 \%$ were less than 5 years of age, $36.09 \%$ were $5-10$ years of age, $20.3 \%$ were $10-15$ years and $24.81 \%$ were more than 15 years (Table 2). and was $71.42 \%$ in patients presented from district Swat.

All the patients responded well to the antibiotics prescribed after culture reports and majority of them recovered without any complications. $11.6 \%$ patients had enteric hepatitis, $4.6 \%$ patients had pancytopenia and one patient received in septic shock and was managed with meropenem and discharged. No deaths were reported. The increase in MDR and XDR-typhoid fever is an issue of discussion here as there are very few drugs and soon there will be no option to treat typhoid fever.

\section{DISCUSSION}

In our study, we reported a high resistance to first line drugs i.e. chloramphenicol $83.72 \%$, ampicillin $75.58 \%$, co-trimoxazole $72.09 \%$ and a bit higher resistance rates compared to a study from Aga khan in 2014 i.e. chloramphenicol (66.8\%), ampicillin $(66.1 \%)$, trimethoprimsulphamethoxazole $(66.5 \%)^{14}$. In an Indian study, they reported resistance pattern to MDR typhoid as chloramphenicol $76.6 \%$, amoxicillin $38.3 \%$ and co-trimoxazole $19.1 \%{ }^{15}$.

Resistance to other drugs like Cefoperazone/Sulbactum was $77.90 \%$, Piperacillin/Tazobactam $65.11 \%$. Resistance reported to ceftriaxone was $91.86 \%$ in comparison to various hospitals in Pakistan whose reported different resistant rates in the previous 10 years for ceftriaxone i.e. $92 \%$ from Hyderabad ${ }^{16}, 69.5 \%$ from Karachi ${ }^{17}$, 43.4\% from Punjab ${ }^{18}$ and $28 \%$ from Islamabad $^{19}$. A study from India reported $23.4 \%$ resistance to ceftriaxone ${ }^{15}$.

Fluoroquinolone resistant rate of salmonella typhi was $83.72 \%$ and it was much high compared to $64.1 \%$ reported in a study in 2006 from Aga khan university ${ }^{9}$ and low as compared to another study in 2014 i.e. $88.2 \%^{14}$. This resistance pattern fluroquinolones were very high as compared to a study from India they reported resistance to ciprofloxacin of $25.5 \% 17^{15}$. The frequent use of fluroquinolones in epidemics of MDR salmonella after 1990 as a first line drug created a high resistance also over the counter use of ciprofloxacin for the treatment of enteric fever is most likely the cause ${ }^{8}$.

XDR typhoid is becoming serious threat due to its spread and now its transmission across the globe will risk further lives in terms of morbidity and mortality. Recently United Kingdom, America ${ }^{20}$ and Canada $^{21}$ also reported cases of XDR.

In the era of antibiotic resistance, the globe is facing a real threat in the form of new resistant mutated bugs and a limited option to treat them with drugs. Salmonella typhi is one of the notorious bugs among many more recently hitting the news with its highest spectrum of resistance pattern causing a serious distress to the community and need a prompt and aggressive approach. In a developing country like Pakistan such resistant organisms are public health emergency.

\section{CONCLUSION}

In the light of our study and the highest resistance to the second line anti typhoid antibiotic i.e. $3^{\text {rd }}$ generation cephalosporins and Azithromycin, it is necessary to rationalize the use of antibiotics in acute febrile illness and to educate the community for vaccination against salmonella typhi. Blood culture should be the gold standard test for diagnosis of enteric fever. XDR-typhoid is on the rise in Pakistan and needs immediate and necessary steps to prevent it. 


\section{REFERENCES}

1. Harris JB, Brooks WA. Typhoid and paratyphoid (Enteric) fever. In: Hunter's Tropical Medicine and Emerging Infectious Disease. Elsevier; 2013. p. 568-76.

2. Dougan G, Baker S. Salmonella enterica serovar Typhi and the pathogenesis of typhoid fever. Annu Rev Microbiol. 2014;68:317-36.

3. Stanaway JD, Reiner RC, Blacker BF, Goldberg EM, Khalil IA, Troeger CE, et al. The global burden of typhoid and paratyphoid fevers: a systematic analysis for the Global Burden of Disease Study 2017.

4. Ochiai RL, Acosta CJ, Danovaro-Holliday MC, Baiqing D, Bhattacharya SK, Agtini MD, et al. A study of typhoid fever in five Asian countries: disease burden and implications for controls. Bull World Health Organ. 2008;86(4):260-8.

5. Kothari A, Pruthi A, Chugh TD. The burden of enteric fever. The Journal of Infection in Developing Countries. 2008 Aug 1;2(04):253-9.

6. Ali A, Ali HA, Shah FH, Zahid A, Aslam H, Javed B. Pattern of antimicrobial drug resistance of Salmonella Typhi and Paratyphi A in a Teaching Hospital in Islamabad. J Pak Med Assoc. 2017;67(3):375-9.

7. Chawla V, Bhujwala RA, Chandra RK, Ghai OP. Typhoid fever in children. Clinical and bacteriological studies. Indian J Pediatr. 1970;37(269):239-44.

8. Walia M, Gaind R, Mehta R, Paul P, Aggarwal P, Kalaivani M. Current perspectives of enteric fever: a hospital-based study from India. Ann Trop Paediatr. 2005;25(3):161-74.

9. Hasan R, Zafar A, Abbas Z, Mahraj V, Malik F, Zaidi A. Antibiotic resistance among Salmonella enterica serovars Typhi and Paratyphi A in Pakistan (20012006). J Infect Dev Ctries [Internet]. 2008;2(04). Available from: http://dx.doi.org/10.3855/jidc.224

10. Klemm EJ, Shakoor S, Page AJ, Qamar FN, Judge K, Saeed DK, et al. Emergence of an extensively drugresistant Salmonella enterica serovar Typhi clone harboring a promiscuous Plasmid encoding resistance to fluoroquinolones and third-generation cephalosporins. MBio [Internet]. 2018;9(1). Available from: http://dx.doi.org/10.1128/mbio.00105-18

11. Kumar A, Pandit V, Shetty S, Rao CR, Pattanshetty S, Samarasinghe CM. Study of clinical profile and antibiotic sensitivity pattern in culture-positive typhoid fever cases. Indian J Community Med. 2012;37(4):2568.

12. Voysey M, Pant D, Shakya M, Liu X, Colin-Jones R, Theiss-Nyland $\mathrm{K}$, et al. Under-detection of blood culture-positive enteric fever cases: The impact of missing data and methods for adjusting incidence estimates. PLoS Negl Trop Dis. 2020;14(1):e0007805.

13. World Health Organization. Typhoid Feverlslamic Republic of Pakistan. 2018.

14. Qamar FN, Azmatullah A, KaziAM, Khan E, Zaidi AKM. A three-year review of antimicrobial resistance of Salmonella enterica serovars Typhi and Paratyphi A in Pakistan. J Infect Dev Ctries. 2014;8(8):981-6.

15. Patel SR, Bharti S, Pratap CB, Nath G. Drug resistance pattern in the recent isolates of Salmonella Typhi with special reference to cephalosporins and azithromycin in the Gangetic plain. J Clin Diagn Res. 2017;11(6):DM013.
16. Qamar FN, Yousafzai MT, Khalid M, Kazi AM, Lohana H Karim S, et al. Outbreak investigation of ceftriaxoneresistant Salmonella enterica serotype Typhi and its risk factors among the general population in Hyderabad, Pakistan: a matched case-control study. Lancet Infect Dis. 2018;18(12):1368-76.

17. Anwar T, Rais H, Jamil MF, Safdar S,Amir MR, AltafA, et al. Extended drug resistance in children with typhoid fever. prof med j. 2020;27(03):581-7.

18. Saeed M, Rasool MH, Rasheed $F$, Saqalein M, Nisar MA, Imran AA, et al. Extended-spectrum betalactamases producing extensively drug-resistant Salmonella Typhi in Punjab, Pakistan. J Infect Dev Ctries. 2020;14(2):169-76.

19. Ali A, Naeem F, Syed RI. Cephalosporin resistance in typhoid and paratyphoid infections-an alarming situation. Journal of Medical Sciences. 2020 Apr $21 ; 28(1): 3-5$

20. Ghizzone M. XDR typhoid infections in 5 US children tied to Pakistan outbreak. Infectious Diseases in Children. 2019 Feb 1;32(2):18-23.

21. Wong W, Al Rawahi H, Patel S, Yau Y, Eshaghi A, Zittermann S, Tattum L, Morris SK. The first Canadian pediatric case of extensively drug-resistant Salmonella Typhi originating from an outbreak in Pakistan and its implication for empiric antimicrobial choices. IDCases. 2019 Jan 1;15:e00492.
DATA SHARING STATEMENT: The data that support the findings of this study are available on request from the corresponding author. The data are not publicly available due to privacy or ethical restrictions.

CONFLICT OF INTEREST: Authors declared no conflict of interest.

GRANTED SUPPORT AND FINANCIAL DISCLOSURE: Nil

\section{AUTHOR'S CONTRIBUTION}

Following authors have made substantial contributions to the manuscript as under

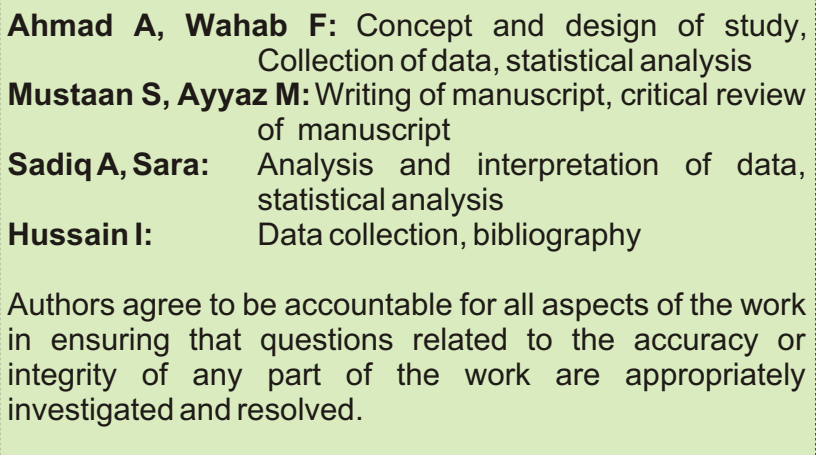

